tumor mass $>2 \mathrm{~cm}(P<0.0001)$, and tumor platinum resistance $(P<0.001)$, but it was not associated with tumor histologic grade and type, patient age, or preoperative serum cancer antigen 125 level. Patients with elevated serum CRP levels had lower overall 5-year survival than those with normal $(\leq 1 \mathrm{mg} / \mathrm{dl})$ serum CRP levels $(58.5 \%$ vs $82.0 \% ; P<0.001)$. Serum CRP was independently associated with overall 5-year survival in multivariate analysis (hazard ratio 1.05; $P<0.001$ ), but it was not predictive of survival in a univariate analysis of a small subgroup of patients with stage I tumors.

The authors conclude that preoperative serum CRP level could potentially serve as a cheap and easily obtained marker of prognosis in patients with ovarian cancer.

Original article Hefler LA et al. (2008) Serum C-reactive protein as independent prognostic variable in patients with ovarian cancer. Clin Cancer Res 14: 710-714

\section{Nomograms for predicting survival of patients with glioblastoma}

In a previous trial conducted by the European Organisation for Research and Treatment of Cancer (EORTC) and the National Cancer Institute of Canada (NCIC) Clinical Trials Group, it was shown that adjuvant temozolomide and radiotherapy can significantly increase survival time for patients with glioblastoma. Gorlia et al. conducted an exploratory subanalysis of data from this trial to investigate the prognostic factors for survival in patients with glioblastoma and to construct nomograms for the prediction of median survival times and 2-year survival probabilities for these patients.

The analysis was based on the survival data, updated in September 2006, of 573 patients who had been newly diagnosed with glioblastoma and had been randomly assigned to standard radiotherapy alone or to radiotherapy plus adjuvant temozolomide as part of the EORTC-NCIC trial. Multivariate analysis showed that treatment with temozolomide, younger age, more-extensive tumor resection, a Mini-Mental State Examination score of $\geq 27$, and no corticosteroid treatment at baseline were independently positively associated with both longer median survival time and a higher 2-year survival probability. For those patients who underwent radiotherapy plus adjuvant temozolomide, improved WHO performance status and the presence of a methylated $\mathrm{O}^{6}$-methylguanine-DNA methyltransferase (MGMT) promoter were also associated with improved survival.

The authors incorporated these factors into nomograms, which can provide tailored prognostic information for individual patients. The nomograms developed can be found at http://www.eortc.be/tools/gbmcalculator.

Original article Gorlia T et al. (2008) Nomograms for predicting survival of patients with newly diagnosed glioblastoma: prognostic factor analysis of EORTC and NCIC trial 26981-22981/CE.3. Lancet Oncol 9: 29-38

\section{Sequential versus concomitant chemotherapy and tamoxifen in breast cancer}

Chemotherapy is generally administered before hormone therapy in patients with endocrineresponsive breast cancer, but there is a paucity of data to indicate that long-term outcomes are better with this regimen than if the two therapies are administered concomitantly.

Del Mastro et al. retrospectively analyzed the importance of the timing of chemo-endocrine therapy by assessing data from a subgroup of patients with surgically treated hormonereceptor-positive breast cancer who were enrolled in two randomized phase III trials of adjuvant anthracycline-based chemotherapy and tamoxifen. Patients who had received tamoxifen within 23 days of the first cycle of chemotherapy were analyzed as the concomitant group, and those who started tamoxifen after chemotherapy or $\leq 7$ days before the last cycle of chemotherapy were assigned to the sequential group.

There was no significant difference in overall survival or event-free survival (time to local or distant relapse, contralateral primary cancer or death from any cause) between the concomitant group $(n=507)$ and the sequential group $(n=589)$, or in cumulative 5-year and 10-year measures of these outcomes. Comparison of sequential versus concomitant therapy in various subgroups showed that there were decreasing trends in hazard ratio with increasing age for both overall and event-free survival $(P=0.015$ and $P=0.063$, respectively).

The authors conclude that concomitant administration of chemotherapy and tamoxifen 